\title{
Resource allocation and interference management for multi-layer wireless networks in heterogeneous cognitive networks
}

Yun Meng ${ }^{*}$ and Xinyi Liu

\begin{abstract}
Aiming at the research of resource allocation and interference management in multi-layer wireless networks in heterogeneous cognitive networks, this paper mainly discusses the resource allocation and interference management in heterogeneous cognitive networks and proposes our proposed cluster-based cooperative interference management scheme. This scheme minimizes the cross-layer interference of multiple base station cells to primary users and avoids the same-layer interference between base station cells through joint channel allocation and power allocation. Through cognitive perception or joint scheduling, the available channel can be obtained by the home base station cell, and then the channel, power and other resources can be allocated according to the related algorithms proposed by us, so as to ensure the harmonious coexistence of the home base station cell and the authorization system such as macrocell. Finally, we validate the proposed algorithm. The simulation results show that the algorithms are effective in reducing interference, but the degree is different.
\end{abstract}

Keywords: Heterogeneous cognition, Wireless network, Resource allocation, Interference management

\section{Introduction}

In recent years, various wireless communications and network technologies have been developing rapidly. Cognitive radio network, small cell network, wireless local area network, WiMAX, and LTE network will promote the next generation network communication technology to achieve greater development. In addition, tablet computers, smartphones, and so on have gradually become an indispensable office and communication tools in people's daily life [1-3]. With the popularity of these new handheld devices, data-intensive applications such as video conversation, online games, online music, and video streaming are becoming more and more popular, and the era of mobile Internet has come. In order to provide a higher quality of service and better user experience, future mobile wireless communication network requires a higher data rate $[4,5]$. Home base station cellular network is a typical representative of small cellular

\footnotetext{
* Correspondence: jpji24@163.com

Chang'an University, Xi'an 710064, China
}

network, usually covering a home or an office area, and has many advantages such as low power consumption, low cost, and plug and play. However, the scarcity of spectrum resources and the interference with macrocell is one of the key problems to be solved before largescale deployment of home base station cells [6-8]. Cognitive radio technology can be applied to the cellular network of home base stations to weaken interference.

In addition to cross-layer interference with macrocell networks, there is still a layer of interference between cellular networks of home base stations. Moreover, in intensive deployment, this same level interference will become very serious. The traditional solution is graphbased coloring. This technology is commonly used in channel allocation in wireless networks to avoid thousands of interference. However, in the case of intensive deployment, due to the lack of sufficient channel resources, it may not be possible to apply coloring technology to effectively solve the same layer interference problem [9-12]. At the same time, due to the relatively small coverage of the base station cell, the same-layer 
interference often appears as a localization phenomenon, and CR technology can play a key role in obtaining such local interference information, including perception, processing, decision-making, and so on. Interference control and power allocation in cognitive radio networks have their own characteristics, and many scholars have conducted an in-depth study on this. However, for interference management based on power control in wireless heterogeneous networks, many studies only try to solve one of the thousand disturbances in the same layer or across layers, assuming that the other interference has been well solved [13-15]. Therefore, it is still very urgent and necessary to combine CR technology with power control and explore a reasonable resource allocation method to solve cross-layer interference and co-layer interference simultaneously.

\section{Related work}

The research contents of this paper mainly include two aspects: research and analysis of the television frequency band white space and the use of cognitive network using the television frequency band white space interference control. This paper proposes a resource allocation and interference management scheme based on cognitive radio in heterogeneous networks. It also solves the cross-layer interference between home base station cellular networks and macrocellular networks and the same-layer interference between home base station cellular networks and realizes the rational allocation and optimization of resources [16]. How to deal with the 1000-disturbance management problem under the heterogeneous network architecture is directly related to the feasibility of adopting this architecture in the future $5 \mathrm{G}$ system. Cross-layer interference management in heterogeneous networks is an important research topic in theory, technology, and application. It is of great theoretical significance and practical value to study key technologies such as resource allocation and interference management in heterogeneous networks. Therefore, this paper closely follows the academic research frontier, from the perspective of green communication, combined with optimization and game theory tools, in-depth study of resource allocation, and interference management algorithms in heterogeneous network systems.

The emergence of new high-end handheld devices such as smartphones and tablets irreversibly shifts users' attention to more data-intensive applications, such as online games, video sessions, online audio, video streaming, etc. Research shows that this fast-growing demand for high data rate comes mainly from indoor environment. The indoor radio coverage is relatively weak due to the penetration loss of the building wall, especially when the user is at the edge of the cell, away from the macro base station to provide services, which an adverse phenomenon is more obvious. In indoor environment, there is a significant mismatch between high data rate requirement and low receive signal-to-interference-plusnoise ratio (SINR) $[17,18]$. Increasing the power of the base station's transmitted signal does not solve the problem, because it will lead to more serious co-channel interference to neighboring cells. Similarly, increasing the number of base stations to achieve better signal coverage is not feasible, because base station deployment costs a lot. In this case, the wireless heterogeneous network composed of the home base cell and the macrocell arises at the historic moment.

Home base station cellular network is a low-cost, plug-and-play network system, mainly used to extend radio coverage to indoor environment, so it can support rapidly increasing data rate requirements. The home base station cellular network consists of a family base station and several mobile stations. This base station is also called home node B or home cellular network access point. Usually, home base station cellular networks are deployed in macrocellular systems in the form of underlay or overlay [19]. Because the home base station is located indoors, the mobile station can connect it directly without having to connect the outdoor macro base station so that all network traffic is transmitted back and forth through wired subscriber digital lines, passive optical networks, or a dedicated wireless channel.

The deployment of home base cell cellular network can be of great help to network operators and users. Due to short-range communication, usually less than 10 $\mathrm{m}$, the link between transceivers is robust, so high-order modulation can achieve a high data rate. Moreover, the indoor mobile station is switched from the Acer station to the family base station, thus releasing many channel resources. In addition, since mobile stations can now be connected to a close home base station rather than a long-distance macro base station, they can communicate at lower transmit power. In terms of battery life for wireless devices, this means significant energy savings, which are critical for wireless devices. An additional benefit of low transmit power is that it increases the probability of spectrum reuse, which correspondingly increases the capacity of the entire system.

Although the home base station cellular is an attractive solution, there are still many technical, protocol, or economic obstacles to deployment. Among them, interference management is one of the most important technical challenges in the deployment of home base station cellular network, which includes the management of cross-layer and inter-layer interference [20,21]. Because the home base station cell is deployed by the end-user, the pre-deployed network planning is not feasible. This uncooperative deployment can lead to serious interference between the home base station cells, especially in 
intensive deployment scenarios. Another challenge is to manage seamless switching between macro and home base stations and between different home base stations. In addition, timing, synchronization, and support for quality of service (QoS) guarantee and portable management of home base stations are urgent problems to be solved.

\section{Methods}

Home base station cell is mainly designed to extend the voice data and other services provided by the macrocell to the indoor environment, so the key design requirement of the network is to ensure that the home base station cell provides the same reliable services as the macrocell. As mentioned earlier, in order to ensure high data rate services, traffic is transmitted through wired networks. In order to ensure coexistence with macrocellular system and ensure smooth communication through reasonable management switching mode, there must be a wired communication path between macrocellular system and home base station cellular network [22].

A typical cellular network architecture of the home base station is shown in Fig 1. Each home base station is connected to a local home base station cellular gateway (fusion gas welding (FGW)). The gateway provides security-related support such as authentication, encryption, and authentication for each home base station cell and is the interface between the home base station peak nest and the core macrocellular network. According to the difference of radio resource management schemes, the cellular gateway of home base station also undertakes certain resource allocation and control functions. For example, the Radio Network Controller (RNC) can share information about spectrum allocation for macrocellular users at specific locations with the local home base station cellular gateway to inform nearby fixed action patterns (FAPs) to avoid using these spectrum resources.

FGW can replace RNC to perform various management and control functions, including authentication and IP security. It is equivalent to an aggregator that connects physically distributed FAP groups and performs joint resource allocation and traffic scheduling by taking into account the capacity of the FAP and the return network. In addition, the location of the FGW is also important because routing optimization is needed to avoid redundant communication if it is too far from the FAP that connects it.

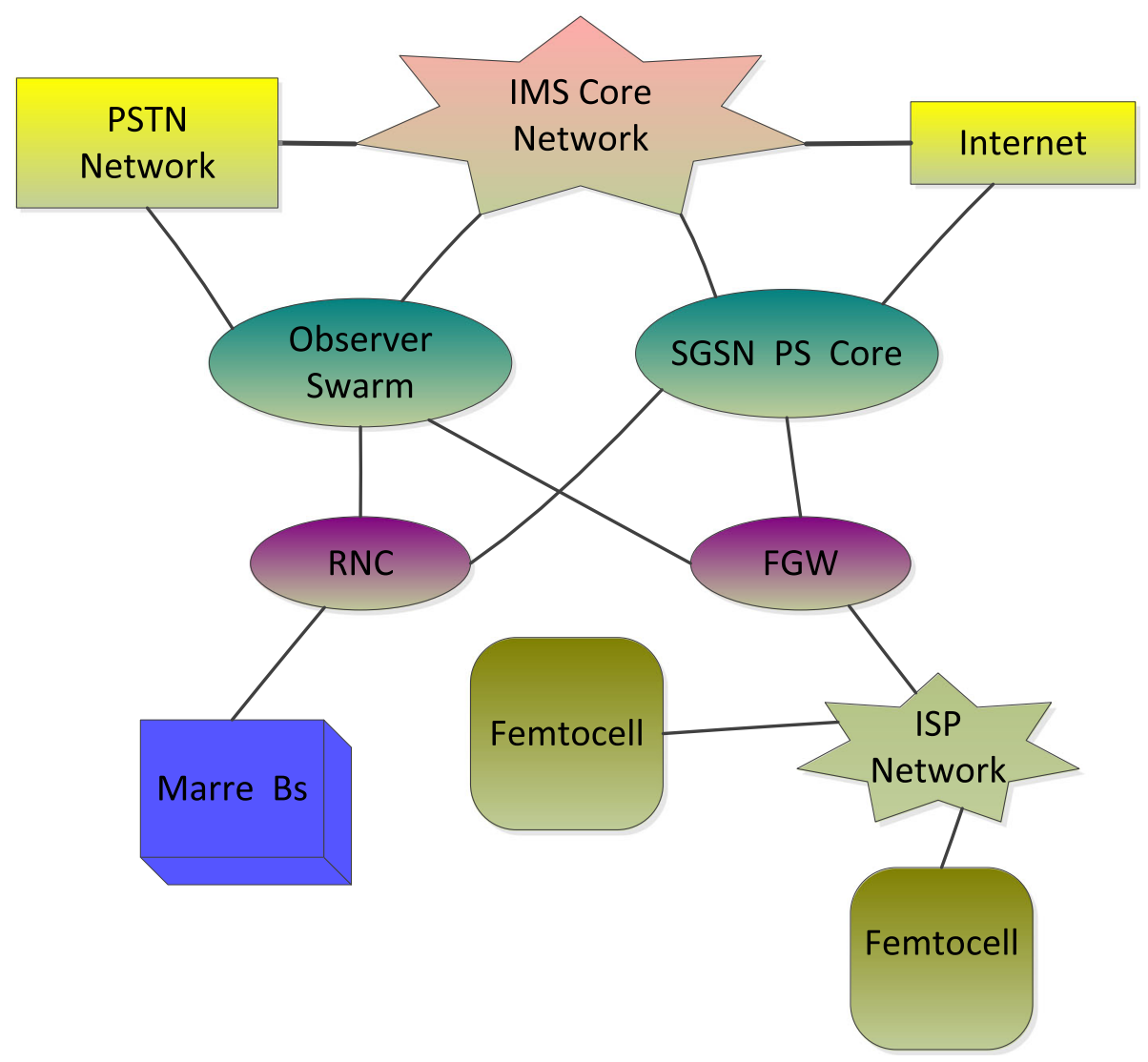

Fig. 1 Network architecture of base station cellular deployment 
3.1 Separation coverage and stacking overlay architecture for home base cellular networks

In the joint deployment of macrocell and home base station cell, macrocell users usually have priority access to spectrum. To ensure harmonious coexistence between the two systems, the home base station cell can also access these spectrums, usually in two modes, namely separate coverage and cascade coverage.

In the separate coverage architecture, the base station cell can share the same spectrum resources as the macrocell, but the base station cell cannot exceed a predefined interference power threshold. This threshold is usually set by the spectrum owner or tenant, with stringent conditions that do not cause harmful interference to the primary user equipment. In this scheme, the cellular network of the home base station is only responsible for maintaining the threshold set by the macrocell [23]. Depending on the policies and regulations, the macrocellular network can also assist in the spectrum allocation decision process. Macrocell can maintain a geographic spectrum occupancy database and share this information with the base station cell via FGW, so that all connected FAPs can be updated as soon as possible under normal or necessary circumstances.

Because the deployment scale of the cellular network of the home base station may be large, and the surrounding radio environment is also changing dynamically, the centralized control of the home base station cell will be very difficult. Hybrid system control seems to be more suitable for home base station cellular network management, where some functions are centralized and others are handed over to FAP or local FGW. In order to facilitate local decision-making, FAP must have an intelligent or cognitive ability. Cognitive home base station cells require periodic perception of the environment to detect the presence of new macrocellular subscribers and any changes in the radio environment. However, the main drawback of this perception-based system is that FAP and users require additional energy and resources to share information.

In the cascade overlay architecture, the home base station cellular network can share the same spectrum resources with the macrocell, but the spectrum used by the macrocell and the home base station cell must be interlaced. Where macrocellular users have priority, home base station cellular users cannot use the spectrum being used by nearby macrocellular users. On the other hand, for geographic database information sharing and spectrum allocation based on perception, separate coverage and cascade coverage systems are very similar.

No matter what kind of deployment plan it is, it is very important to solve the problem of whether the channel is occupied. Improper decision-making can lead to false alarm invalidation, that is, when a channel is actually occupied, it is detected that it is not occupied. This will cause great harm to other users. Conversely, if a channel is not occupied but it is mistakenly detected that it is occupied, the channel will be idle, resulting in waste. In order to make effective judgments, the level of interference generated at any location needs to be accurately predicted or measured.

In fact, the amount of interference received by a cellular phone user is a function of distance. The path loss (PL) component can be calculated using the following models:

$$
\begin{aligned}
\mathrm{PL}^{m}= & 128+37.6 \log \left(\frac{d}{1000}\right)+21 \log \left(\frac{f_{c}}{2}\right) \\
& +L_{\mathrm{WP}} \\
\mathrm{PL}_{\mathrm{LOS}}^{f}= & 18.7 \log (d)+46.8+20 \log \left(\frac{f_{c}}{5}\right) \\
\mathrm{PL}_{\mathrm{NLOS}}^{f}= & 20 \log (d)+46.4+20 \log \left(\frac{f_{c}}{5}\right) \\
& +L_{\mathrm{WP}}
\end{aligned}
$$

In which, $\mathrm{PL}^{m}$ is the path loss component of macrocell, $d$ is the distance from macrocell base station, in meters, $f_{c}$ is the carrier frequency, $L_{\mathrm{WP}}$ is the wall penetration loss, and $\mathrm{PL}_{\mathrm{LOS}}^{f}$ and $\mathrm{PL}_{\mathrm{NLOS}}^{f}$ are the path loss of both LOS and non-LOS signals. The farther away from the Acer station, the smaller the interference from the cellular users of the home base station is. When the distance reaches a certain value, the interference power drops to an acceptable threshold, which is a minimum safe distance. Moreover, the interference level also varies with the transmission power of the macrocell base station. When macro base stations communicate with high power, the minimum safe distance will increase greatly. In summary, by controlling the power of the macro base station, more channel space can be released for use by the home base station cell. The capacity of the whole system can be significantly improved by adopting a joint optimization strategy for peak-to-nest transmission power of macrocell and home base station. Of course, there is a price to pay for this improvement, which is the increased computational complexity and the cost of return communication.

\subsection{Home base station cellular access mechanism: closed- loop, open, and hybrid}

Home base station cellular generally operates in three different access modes, namely, closed-loop, amplifier, and hybrid. Each model has its own merits and demerits. Importantly, the choice of access mechanism not only affects the operation of home base station cell and 
macrocell, but also affects cross-layer interference cancelation and handover management.

In the closed loop access mode, only a fixed number of predefined subscribers is allowed to access the network. An unregistered user cannot access a home base station cell even if he has a strong signal link to a particular FAP. Any unregistered user entering the coverage of a closed-loop home base station cell will suffer severe cross-layer or co-layer interference when he attempts to connect to a macrocell or other home base station cell. Therefore, a closed-loop access system must adopt strategies to eliminate or avoid these two types of interference. Beamforming based on multiple input multiple output (MIMO) antennas is a way to reduce interference. Another alternative technique is to implement a negotiation mechanism between jammed transmitters, which allows them to switch the operating spectrum under strong jamming conditions. The main advantage of this system is that the number of users is limited, and management and accounting are relatively simple.

Compared with the closed-loop access mode, the open access mode allows any user within the FAP coverage to access the network. The advantage of this mode is that the downlink can unload large amounts of traffic from the macrocell to the home base station cell, whereby the macrocell can release several channels for reuse, which is particularly useful at the cellular edges where the macrocell coverage is generally poor. Although saving valuable macrocell resources, the open access system still has many problems. Because of the small coverage, if the user is mobile, the system has to undergo multiple handovers, so innovative design methods are needed to minimize the number of handovers. In addition, the number of unregistered and unregistered visitors has become very high, registered users may encounter traffic congestion in the home base station cell. There are also security issues, because open access systems are inherently vulnerable, such as hacker intrusion, illegal monitoring activities, location detection, malicious node attacks, and so on. Effective measures must be taken to prevent malicious manipulation of embedded software, which will change the location information and so on, hindering network management.

The hybrid access mode combines the advantages of the above two systems. Considering capacity and gain, the hybrid model allows a limited number of unregistered users to gain access to the network as well as existing registered users. This restriction on the number of visitors can achieve significant performance improvements without compromising registered users. In addition, the mechanism that registered users have priority over non-registered users should be introduced to ensure the satisfaction of registered users. Billing differentiation is also an option to consider to distinguish between registered users and non-registered users.

\section{Research on power control and resource} allocation in heterogeneous networks

\subsection{System description}

Considering the two-layer heterogeneous downlink OFDMA system model of deploying flying cells as shown in Fig. 2, a macrocell network base station multiplexes $\mathrm{K}$ orthogonal resource blocks (FRBs) with a frequency reuse factor of 1 . All users or base stations in the system are equipped with single or single antenna omnidirectional antennas. M co-channel deployed beehive densely deployed in macrocell coverage but far away from MBS. MUs are deployed near the flying cell area and away from the MBS serving it. In the downlink transmission scenario of the system, the communication service of the flying cell causes a communication blind area to the macrocell. Therefore, the cross-layer interference power received by MUs from the flying cell must be limited to a certain range to ensure its normal communication quality of service. In addition, due to the intensive deployment and frequency reuse of the flying cell, the same layer interference between the flying cells cannot be ignored. The bandwidth of each resource block in the system is B0. Due to OFDMA technology, all subchannels in communication can be regarded as flat-fading subchannels. In this paper, Rayleigh fading, shadow fading, and path loss are used to model all subchannels.

In this paper, OFDMA technology is used. According to the Shannon capacity formula, the sum rate of the flying honeycomb can be expressed as the following mathematical expression:

$$
C^{(i)}(P, A)=\sum_{n=1}^{N_{i}} \sum_{k=1}^{K} B_{0} a_{n, k}^{(i)} \log _{2}\left(1+\gamma_{n, k}^{(i)}\right)
$$

Correspondingly, the capacity of the entire cellular network can be expressed as:

$$
C(P, A)=\sum_{i=1}^{M} C^{(i)}(P, A)
$$

In which, $P$ represents the transmit power matrix of each FBS, defined as $P=\left[P^{(1)} \ldots P^{(i)} \ldots P^{(M)}\right]$ and $P^{(i)}=[$ $\left.p_{1}^{(i)} \cdots p_{k}^{(i)} \cdots p_{K}^{(i)}\right]$. A represents the allocation indicator matrix of the resource block, which is defined as $A=\left[A^{(1)} \cdots A^{(i)} \cdots A^{(M)}\right]$ and $A^{(i)}=\left[a_{1}^{(i)} \cdots a_{k}^{(i)} \cdots a_{K}^{(i)}\right]$. The $a_{n, k}^{(i)}$ value can only be 0 or 1 , indicating whether the $k$ th resource block is allocated to the $n$th user in the $i$ th flying cell.

In order to carry out energy efficient power control and resource allocation, we need to consider the total power consumption of the system. According to the power loss model in the references, the total power 


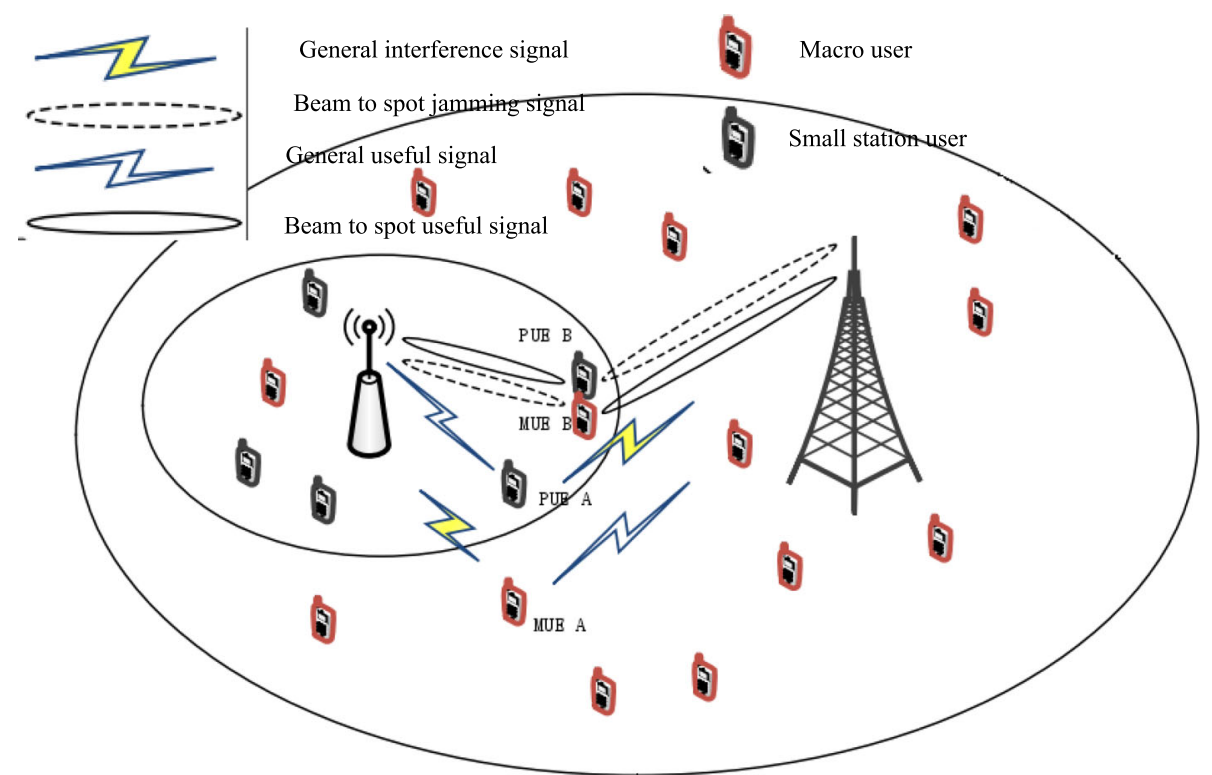

Fig. 2 System scenario model diagram

consumption of the system includes the transmitting power of the power amplifier and the circuit power loss of the FBS terminal. At the same time, in order to effectively design the energy, the channel state information and some parameter information between FU and FBS need to be exchanged, and the power loss of the return link cannot be ignored. Therefore, for the first $i$ cellular cell, its power consumption is

$$
P_{t o t}^{(i)}(P, A)=\phi_{a m} \sum_{n=1}^{N_{i}} \sum_{k=1}^{K} a_{n, k}^{(i)} p_{n, k}^{(i)}+P_{C}^{(i)}+P_{B H}^{(i)}
$$

Correspondingly, the power loss of the whole flying cellular network can be expressed as follows:

$$
P_{t o t}^{(i)}(P, A)=\sum_{i=1}^{M} P_{t o t}^{(i)}(P, A)
$$

In which, $\phi_{a m}$ represents the inefficiency of FBS terminal power amplifier. $P_{C}^{(i)}, P_{B H}^{(i)}$ represents the power loss of the $i$ flying cell and the power loss of the return link.

For the downlink transmission of the network, the flying cell will cause a communication blind area for the coverage of the macrocell. Therefore, it is necessary to design a cross-layer interference power management scheme to protect the communication QoS of MUs distributed near the flying cell. The interference management scheme adopted in this paper is similar to the interference temperature limit in cognitive radio system. The interference management scheme needs to ensure that the cross-layer interference power received on the
MU invoked by MBS is below the preset power threshold Q. Mathematically, the cross-layer interference management plan is as follows:

$$
I_{k}=\sum_{i=1}^{M} \sum_{n=1}^{N_{i}} a_{n, k}^{(i)} p_{n, k}^{(i)} h_{k}^{F_{i} M} \leq Q, \forall k \in\{1,2, \cdots, K\}
$$

In which, $h_{k}^{F_{i} M}$ denotes the channel power gain of the MU invoked by the first FBS to MBS on the K resource block, and $I_{k}$ denotes the total cross-layer interference power received by the MU invoked on the $k$ resource block from the flying cellular network.

\subsection{Simulation results and performance analysis}

Consider a two-tier heterogeneous network environment consisting of three flying cells deployed within the macrocell coverage but away from the MBS. Each flying cell is randomly deployed with three FUs. Some MUs are deployed around the flying cell but away from the MBS. Here, the channel is modeled as a combination of path loss (indoor and outdoor), shadow fading, and fast fading following complex Gaussian distribution $C \mathrm{~N}(0,1)$.

All simulation results are obtained through 500 random channel averages. This paper compares the proposed and non-cooperative energy efficiency schemes with non-cooperative spectrum efficiency schemes, respectively. In order to compare the fairness performance of different schemes, this paper adopts the dimension of fairness defined in the literature: 


$$
F_{\text {airness }}=\frac{\left(\sum_{i=1}^{M} E E_{\text {-avg }}^{(i)}\right)^{2}}{M \sum_{i=1}^{M}\left(E E_{\text {-avg }}^{(i)}\right)^{2}}
$$

In which, $E E_{-a v g}^{(i)}$ represents the average energy efficiency of $i$ flying honeycomb.

Figure 3 shows the relationship between the minimum energy efficiency of a flying cellular system and the cross-layer interference power constraint level Q when the maximum transmission power of FBSs is $P_{\max }=$ $20 \mathrm{dBm}$. It can be seen from the diagram that the energy efficiency fairness scheme of the system is superior to other schemes in the index of the minimum energy efficiency of the flying honeycomb in the system. The global energy efficiency optimal scheme is only in the case of relatively loose interference level, this is because when the level of cross-layer interference is relatively loose, the game participants' strategy space will become larger, each game participant for their own benefit, which will be more unscrupulous to increase its launch power. However, the same layer interference will be increased when the transmitting power is increased, resulting in the decrease of its own rate, which will lead to the decrease of energy efficiency. Later, this paper will show that the global energy efficiency optimization scheme has the highest energy efficiency compared with other schemes. In addition, we find that the minimum energy efficiency of the two schemes increases with the level of cross-layer interference becoming looser and looser, while the other two schemes do not have this trend because of the selfishness of non-cooperative game.

Figure 4 shows the relationship between energy efficiency fairness and cross-layer interference constraint level $Q$ for various schemes when the maximum transmission power of FBSs is $P_{\max }=20 \mathrm{dBm}$. Obviously, the energy efficiency fairness of the system energy efficiency fairness scheme is superior to all other schemes, especially when the level of cross-layer interference is more strict, and this advantage is more obvious, which also verifies the effectiveness of the proposed scheme. Although the superiority of the proposed energy efficiency optimization scheme is not shown in this diagram, we will show its optimal performance in the overall energy efficiency of the system.

\section{Experience}

\subsection{Physical cluster and virtual cluster design for cellular} base station cellular management

The interference power level introduced by the home base station cell mainly depends on the distance from the home base station cell to the other home base station cell and the authorization system, as well as the transmission power of the FAP. Therefore, we can first allocate the available channels of the base station cell according to the distance, and then adjust the power budget of different FAPs to reduce the interference. Because of the lack of enough channels, the traditional coloring method is not very effective. For this reason, we propose the concepts of physical cluster and virtual

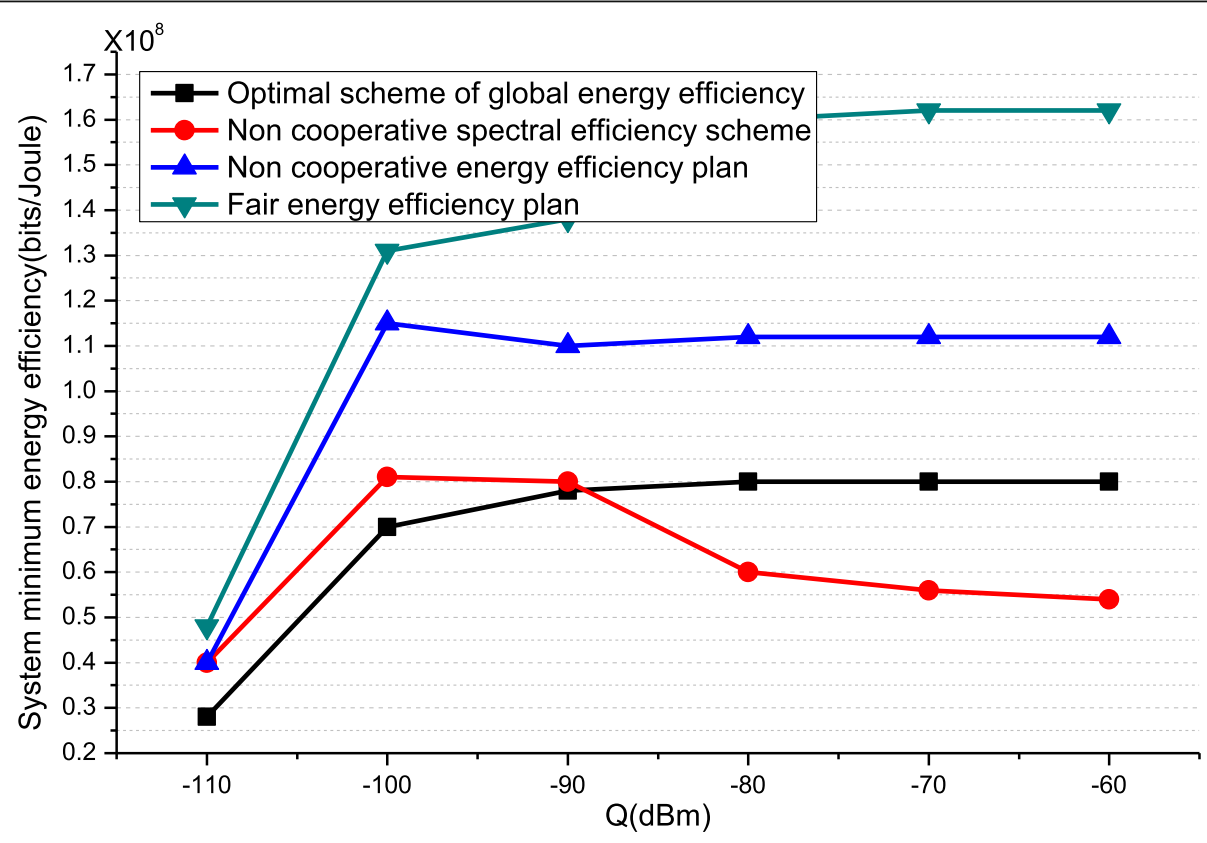

Fig. 3 System minimum energy efficiency vs cross-layer interference constraint Q 


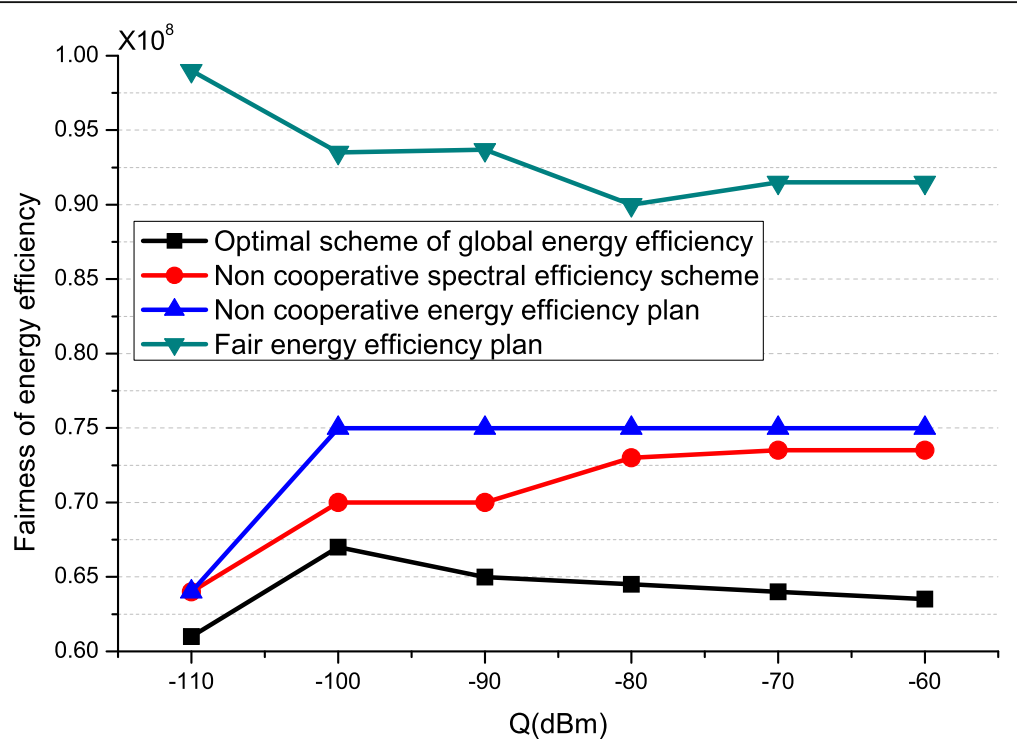

Fig. 4 Energy efficiency fairness vs cross-layer interference constraint Q

family, which are essentially the problem of cellular clustering in home base stations. We define the spatial correlation $\gamma$ of the base station cellular as follows:

$$
\gamma=\frac{r}{d}
$$

Among them, $r$ is the radius of the home base cell cellular coverage and $d$ is the distance between two home base stations. Adjacent home base station cells can be grouped into a physical cluster if and only if the spatial correlation between any two home base station cells satisfies the following constraints:

$$
\gamma \geq \gamma_{0}
$$

Among them, $\gamma_{0}$ is determined by the minimum spatial correlation of safe distance $d_{0}$. Each physical cluster has a cluster center and can be found by some clustering algorithms. In dense deployment scenarios, the number of channels available is very limited, for example, there are only six channels $(1.4 \mathrm{MHz})$. Then, the number of base station cells clustered into a physical cluster cannot exceed six, because the base station cells in the physical cluster need to use different channels to avoid the same layer interference.

The concept of virtual cluster is relative to the physical cluster. The physical cluster is mainly related to the location information of the base station cellular, while the virtual cluster is equivalent to a logical cluster. The home base station cells occupying the same channel but located in different physical families can be grouped into a virtual cluster. However, if two physical clusters are too close apart, the potential harmful co-layer interference is still unacceptable. Therefore, we define the spatial correlation $\gamma$ ' of the physical family as follows:

$$
\gamma^{\prime}=\frac{R}{D}
$$

In which, $R$ represents the radius of the physical family and also depends on the safe distance $d_{0}$. $D$ represents the distance between two physical clusters. In order to ensure that interference between base station cells in the virtual family is tolerable, another constraint is required:

$$
\gamma^{\prime} \leq \gamma_{0}^{\prime}
$$

In which, $\gamma_{0}^{\prime}=R /\left(2 R+d_{0}\right)$. If $R$ is defined as half of $d_{0}$, then $\gamma_{0}^{\prime}=1 / 4$. In other words, the cell members of the home base station occupying the same channel in the virtual cluster need to be separated as far as possible to minimize interference with each other.

\subsection{Simulation and performance analysis}

We assume that there is a thin wall between FAP and secondary users, and there is a thick wall between FAP and main users. Generally speaking, the interference assumption caused by primary user system to secondary users is negligible. The simulation parameters are summarized in Table 1.

Figure 5 shows the performance comparison between the proposed IM algorithm and the other two power allocation algorithms. In this figure, the power budget of FAP is fixed $10 \mathrm{dBm}$. As can be seen from the graph, when the capacity threshold is $10 \mathrm{bit} / \mathrm{s} / \mathrm{Hz}$, the total amount of interference introduced by IM algorithm is approximately one-third of the average power allocation 
Table 1 Simulation parameter settings

\begin{tabular}{ll}
\hline Parameter & Value \\
\hline Coverage radius & $10 \mathrm{~m}$ \\
Maximum number of secondary users & 4 \\
Fixed transmitting power & $10 \mathrm{dBm}$ \\
Minimum transmitting power & $8 \mathrm{dBm}$ \\
Maximum transmitting power & $12 \mathrm{dBm}$ \\
Noise power & $1.4 \mathrm{e}-014 \mathrm{~W}$ \\
Thin wall penetration loss & $5 \mathrm{~dB}$ \\
Thick wall penetration loss & $12 \mathrm{~dB}$ \\
Shadow fading & $6 \mathrm{~dB}$ \\
Carrier frequency & $2 \mathrm{GHz}(600 \mathrm{MHz})$ \\
Channel broadband & $180 \mathrm{KHz}$ \\
Subcarrier broadband & $15 \mathrm{KHz}$ \\
Number of subcarriers per channel & 12 \\
\hline
\end{tabular}

algorithm; when the capacity threshold is increased to $13.4 \mathrm{bit} / \mathrm{s} / \mathrm{Hz}$, the ratio becomes nearly one-half. The effect of IM algorithm on interference reduction is very obvious. In addition, the IM algorithm also outperforms the mentioned residual power average allocation scheme mentioned above. With the increase of capacity threshold, the change of total interference is as follows: the average power allocation algorithm remains unchanged, the average residual power allocation algorithm decreases, and the IM algorithm increases. This is because as the capacity threshold increases, the power allocated to each subcarrier increases and the residual power budget decreases. Therefore, the gap between the residual power average allocation algorithm and the IM algorithm is narrowed. When the residual power budget is reduced to zero, the residual power average allocation algorithm will achieve the same performance as IM algorithm.

Figure 6 shows the aggregate interference curve obtained by using the proposed IM algorithm in a single home base station cell under different power budget constraints. From the graph, we can see that the total amount of interference increases with the increase of capacity threshold and increases with the increase of power budget. In fact, there is a trade-off between the total amount of interference that is minimized and the capacity to maximize. Therefore, it is necessary to select appropriate capacity threshold to control aggregation interference and make it at a certain level to adapt to different situations. In addition, reducing the power budget of FAP can reduce the aggregation interference, but considering the QoS requirements of secondary users, the power budget cannot be too low. In general, the fixed power budget of FAP is around $10 \mathrm{dBm}$.

Figure 7 depicts the impact of the distance from the base station cell to the victim's primary user on the aggregation interference. Obviously, the farther the base station cell is from the victim primary user, the less harmful interference it will cause to the primary user. This is the most direct and effective method of interference mitigation. To meet the interference threshold of the primary user, a single home base station cell must ensure a safe distance. However, when there are multiple home base station cells using the same channel, the secure distance does not apply because the primary user has to ensure that aggregate interference is below the interference threshold. In this case, the home base station cell which is close to the primary user is often limited to communicate with minimal power or jump to other channels.

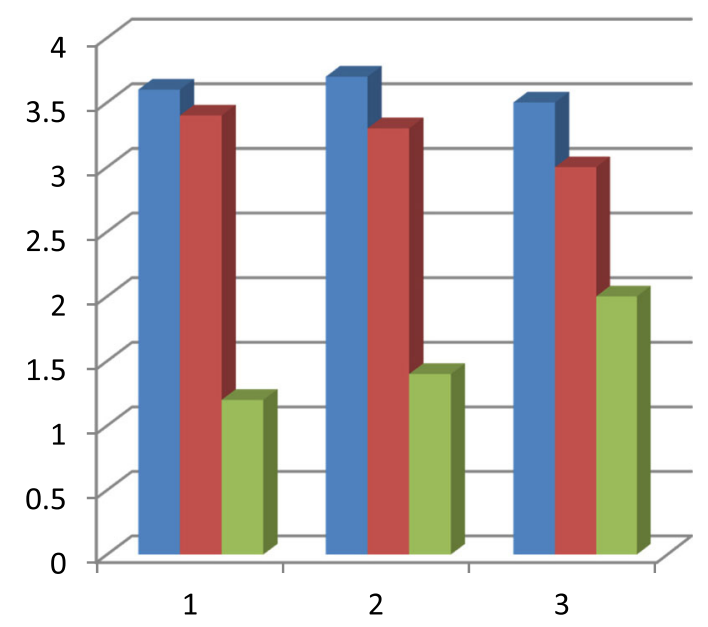

Average Alloction

- Left Fair Alloction

IM Algorithm

Fig. 5 Comparison of different subcarrier power allocation schemes 


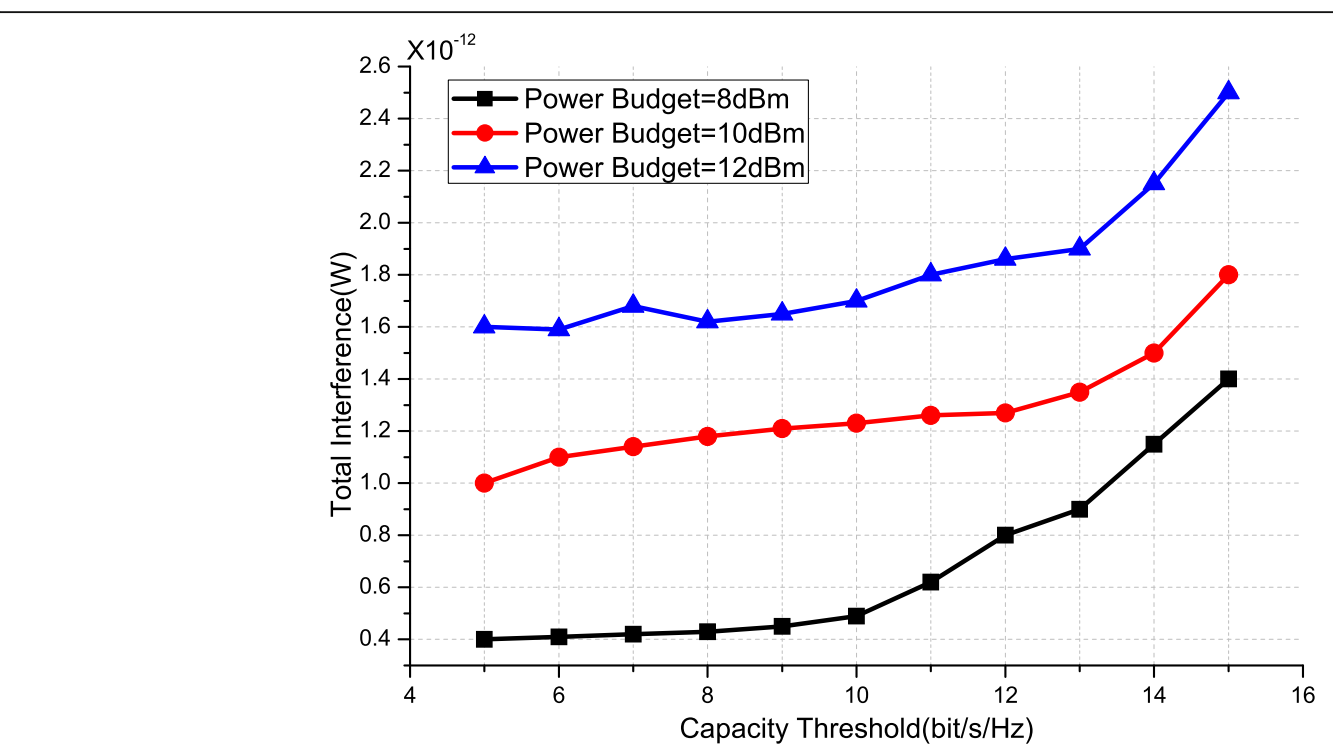

Fig. 6 Aggregation interference under different power budgets

Figure 8 compares the performance of two different frequency bands used by the base station cell. In fact, these two bands represent two typical authorization systems: $2 \mathrm{GHz}$ for macrocellular networks and $600 \mathrm{MHz}$ for television broadcasting systems. Figure 8 shows that under different power budget constraints, the white space of the TV band can meet higher capacity requirements than the macrocellular band. This result should be attributed to the good propagation characteristics of TV frequency bands. At the same time, this indicates that the home base station cell can use the white space of the TV frequency band to obtain high data rate to provide more high-quality services.

\subsection{Results and discussion}

In this paper, two energy efficient power control and resource block allocation schemes are proposed, which are the energy efficiency fairness scheme and global energy efficiency optimization scheme. In this paper, the fractional programming theory and dual decomposition method are used to deal with the non-convex fractional optimization problems of the two schemes. For the system energy efficiency fairness scheme, this paper designs a distributed algorithm with limited cooperation between flying cells to obtain the power control and resource block allocation strategy of approximate fairness of system energy efficiency. The proposed algorithm

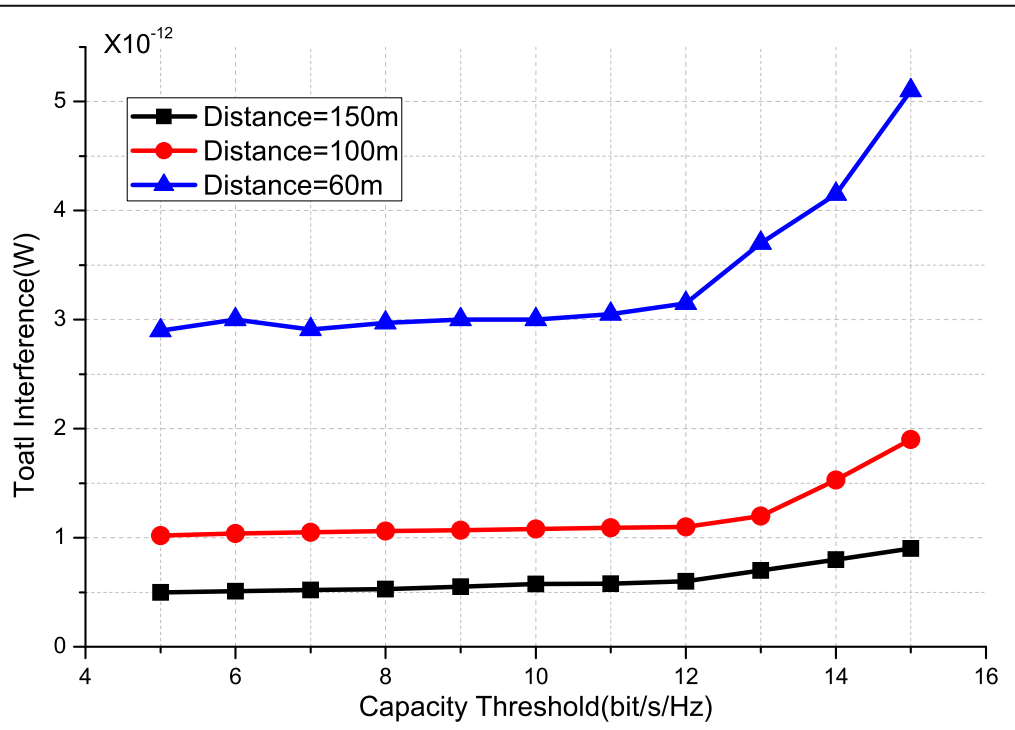

Fig. 7 Aggregation interference at different distances 


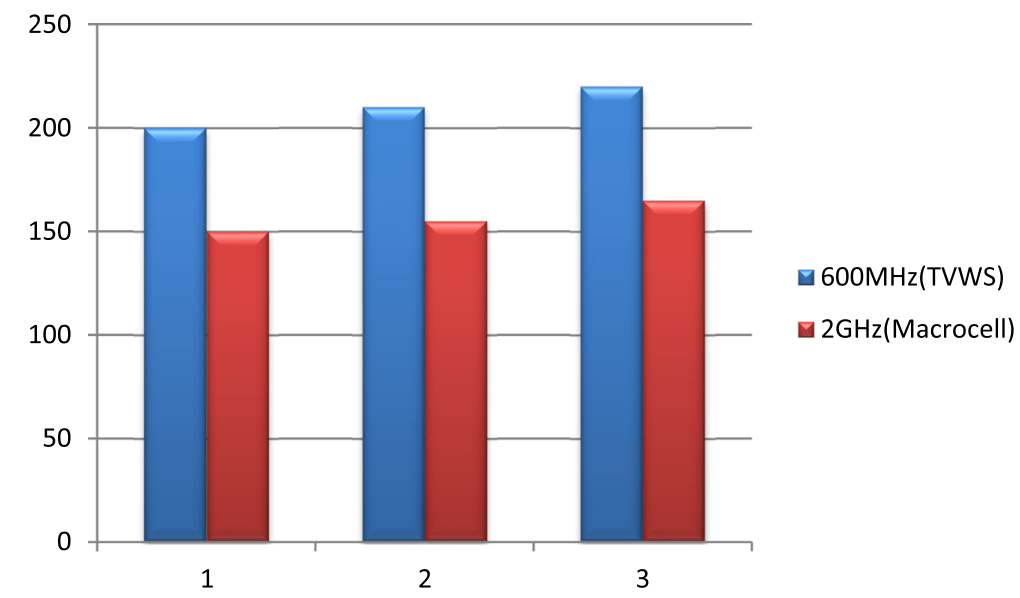

Fig. 8 Comparison of different frequency bands

only needs a small amount of parameter information interaction between FBSs, which reduces the system cooperative communication overhead. Based on the lower bound theory of logarithmic function, this paper transforms the original problem into its lower bound convex optimization problem and designs a heuristic iterative algorithm to approximate the optimal solution of the original problem. Simulation results verify the effectiveness of the proposed two schemes and their superiority in the system minimum energy efficiency, energy efficiency fairness and global energy efficiency index.

\section{Abbreviations}

FAP: Fixed action pattern; FGW: Fusion gas welding; MIMO: Multiple input multiple output; SINR: Signal-to-interference-plus-noise ratio

\section{Authors' contributions}

Yun Meng extablished the model and policy framework, finished the writing of this manuscript. Xinyi Liu carried out the simulation. Both authors read and approved the final manuscript.

\section{Authors' information}

Yun Meng received the $\mathrm{BS}$ and $\mathrm{PhD}$ degrees in communication engineering from Xidian University, Xi'an, China, in 2009 and 2015, respectively. She has been with the School of Electronics and Control Engineering, Chang'an University since 2015. Her main research interests include interference mitigation and radio resource management in heterogeneous networks and graph theory for wireless networks.

Xinyi Liu received his Bachelor degree in communication engineering in 2008, and PhD in communication and information system in 2014 from Xidian University, China. From December 2010 to December 2011, He was a visiting scholar with the Department of Electrical Engineering, Texas A and $\mathrm{M}$ University, USA. Currently, he is an associate professor in the School of Information Engineering, Chang'an University, China. His research interests include Internet of Vehicles, heterogeneous networks, and cognitive radio system.

\section{Funding}

This work was supported in part by the National Natural Science Foundation of China under Grant 61701044 and Grant 61803041, National Key Research and Development Program of China (2018YFB1600600), in part by the Project funded by China Postdoctoral Science Foundation under Grant 2017M623089, Key Research and Development Program of Shaanxi (Program No.2019GY-037), Xi'an Science and Technology Project.

\section{Availability of data and materials}

The datasets used and/or analyzed during the current study are available from the corresponding author on reasonable request.

\section{Competing interests}

The authors declare that they have no competing interests.

Received: 24 March 2019 Accepted: 11 July 2019

Published online: 24 July 2019

\section{References}

1. H. Chen, J. Xu, G.X. Jianglong, Fast auto-clean CNN model for online prediction of food materials. J Parallel Distrib Comput 117(1), 218-227 (2018)

2. K.I. Pedersen, Y. Wang, S. Strzyz, et al., Enhanced inter-cell interference coordination in co-channel multi-layer LTE-advanced networks. IEEE Wirel. Commun. 20(3), 120-127 (2013)

3. F. Likang, H. Ronghui, ICIC and CoMP based interference management scheme in small cell networks. J Xidian Univ 44(5), 32-38 (2017)

4. H. Chen, X. Xie, An efficient recommendation filter model on smart home big data analytics for enhanced living environments. Sensors. 16(10), 1-26 (2016)

5. M. Sheng, J. Liu, X. Wang, et al., On transmission capacity region of D2D integrated cellular networks with interference management. IEEE Trans. Commun. 63(4), 1383-1399 (2015)

6. B. Wu, L. Zong, X. Yan, C. Guedes Soares, Incorporating evidential reasoning and TOPSIS into group decision-making under uncertainty for handling ship without command. Ocean Eng. 164(1), 590-603 (2018)

7. A. Chiumento, S. Pollin, C. Desset, et al., Scalable HetNet interference management and the impact of limited channel state information. Eurasip J Wirel Commun Netw 2015(1), 74-81 (2015)

8. K.V. Rukmani, N. Nagarajan, Enhanced channel allocation scheme for cross layer management in wireless network based on interference management. Clust. Comput. 2018(9), 1-11 (2018)

9. P. Muñoz, D. Laselva, R. Barco, et al., Dynamic traffic steering based on fuzzy Q-learning approach in a multi-RAT multi-layer wireless network. Comput. Netw. 71(3), 100-116 (2014)

10. Y. Wang, E. Zio, X. Wei, D. Zhang, B. Wu, A resilience perspective on water transport systems: The case of Eastern Star. Int J Disaster Risk Reduct 33(1), 343-354 (2019)

11. H. Chen, Y. Ouyang, W. Jiang, An optimized data integration model based on reverse cleaning for heterogeneous multi-media data. Multimed. Tools Appl. 25(1), 1-16 (2015)

12. C. Wan, X. Yan, D. Zhang, Z. Qu, Z. Yang, An advanced fuzzy Bayesian-based FMEA approach for assessing maritime supply chain risks. Transport Res ELog 125(1), 222-240 (2019)

13. M.Y. Zhang, D. Zhang, F. Goerlandt, X. Yan, P. Kujala, Use of HFACS and fault tree model for collision risk factors analysis of icebreaker assistance in icecovered waters. Saf. Sci. 111(1), 128-143 (2018)

14. T. Zhou, C. Wu, J. Zhang, D. Zhang, Incorporating CREAM and MCS into fault tree analysis of LNG carrier spill accidents. Saf. Sci. 96(1), 183-191 (2017) 
15. K. Pandremmenou, L.P. Kondi, K.E. Parsopoulos, A study on visual sensor network cross-layer resource allocation using quality-based criteria and metaheuristic optimization algorithms. Appl. Soft Comput. 26(26), 149-165 (2015)

16. T.M.C. Chu, H. Phan, F.C. Zheng, Physical-layer network coding with multiantenna transceivers in interference limited environments. IET Commun. 10(4), 363-371 (2016)

17. D. Ma, M. Ma, Network selection and resource allocation for multicast in HetNets. J Netw Comput Appl 43(5), 17-26 (2014)

18. C. Jiang, Y. Shi, X. Qin, et al., Cross-layer optimization for multi-hop wireless networks with successive interference cancellation. IEEE Trans. Wirel. Commun. 15(8), 5819-5831 (2016)

19. M. Peng, C. Wang, J. Li, et al., Recent advances in underlay heterogeneous networks: interference control, resource allocation, and self-organization. IEEE Commun Surv Tutorials 17(2), 700-729 (2015)

20. Y. Yu, E. Dutkiewicz, X. Huang, et al., Downlink resource allocation for next generation wireless networks with inter-cell interference. IEEE Trans. Wirel. Commun. 12(4), 1783-1793 (2013)

21. W. Feng, S. Feng, Y. Ding, et al., Prediction-based cross-layer resource allocation for wireless multi-hop networks with outdated CSI. IEICE Trans Commun 97(4), 746-754 (2014)

22. Z. Kaleem, B. Hui, K.H. Chang, QoS priority-based dynamic frequency band allocation algorithm for load balancing and interference avoidance in 3GPP LTE HetNet. Eurasip J Wirel Commun Netw 2014(1), 185-192 (2014)

23. B. Wu, X. Yan, Y. Wang, C. Guedes Soares, An evidential reasoning-based CREAM to human reliability analysis in maritime accident process. Risk Anal. 37(10), 1936-1957 (2017)

\section{Publisher's Note}

Springer Nature remains neutral with regard to jurisdictional claims in published maps and institutional affiliations.

\section{Submit your manuscript to a SpringerOpen ${ }^{\circ}$ journal and benefit from:}

- Convenient online submission

- Rigorous peer review

- Open access: articles freely available online

- High visibility within the field

- Retaining the copyright to your article

Submit your next manuscript at $\boldsymbol{\nabla}$ springeropen.com 